\title{
DIFFERENTIABLE STRUCTURES ON A GENERALIZED PRODUCT OF SPHERES
}

\section{SAMUEL OMOLOYE AJALA}

School of Mathematics
The Institute for Advanced Study and
Princeton, New Jersey 08540

School of Mathematics Princeton, New Jersey 08540
Mathematics Department

University of Lagos

Akoka-Yaba

Lagos-Nigeria, West Africa

(Received April 21, 1986)

ABSTRACT. In this paper, we give a complete classification of smooth structures on a generalized product of spheres. The result generalizes our result in [1] and R. de Sapio's result in [2].

KEY WORDS AND PHRASES. Differential structures, product of spheres. 1980 NMS SUBJECT CLASSIFICATION CODE. 57R55

\section{INTRODUCTION}

In [2] a classification of smooth structures on product of spheres of the form $s^{k} \times s^{p}$ where $2 \leq k \leq p, k+p \geq 6$ was given by $R$. de Sapio and in [1] this author extended $R_{0}$ de Sapio's result to smooth structures on $s^{p} \times s^{q} \times s^{r}$ where $2 \leq p \leq q \leq r$. The next question is, how many differentiable structures are there in any arbitrary product of ordinary spheres. In this paper, we give a classification under the relation of orientation preserving diffeomorphism of all differentiable structures of spheres $s^{k_{1}} \times s^{k_{2}} \times \ldots \times s^{k_{r}}$ where $2 \leq k_{1}<k_{2} \leq \ldots \leq k_{r} \cdot s^{n}$ denotes the unit $n$-sphere with the usual differential structure in the Euclidean $(n+1)-s p a c e R^{n+1}$. $\theta^{n}$ denotes the group of h-cobordism classes of homotopy $n$-sphere under the connected sum operation. $\Sigma^{n}$ will denote an homotopy n-sphere. $H(p, k)$ denotes the subset of $\theta^{\mathrm{P}}$ which consists of those homotopy $\mathrm{p}$-sphere $\Sigma^{\mathrm{P}}$ such that $\Sigma^{\mathrm{P}} \times \mathbf{s}^{\mathrm{k}}$ is diffeomorphic to $s^{\mathrm{P}} \times \mathrm{s}^{\mathrm{k}}$. By [2], $\mathrm{H}(\mathrm{p}, \mathrm{k})$ is a subgroup of $\theta^{\mathrm{P}}$ and it is not always zero and in fact in [1], we showed that if $k \geq p-3$, then $H(p, k)=\theta^{p}$.

By Hauptremutung [3], piecewise linear homoemorphism will be replaced by homeomorphism. Consider two manifolds $s^{k_{1}} \times s^{k_{2}} \times s^{k_{3}} \times s^{k_{4}}$ and $\Sigma^{k_{2}+k_{4}} \times s^{k_{1}} \times s^{k_{3}}$, we shall denote the connected sum of the two manifolds along a $k_{2}+k_{4}-1$ cycle by 


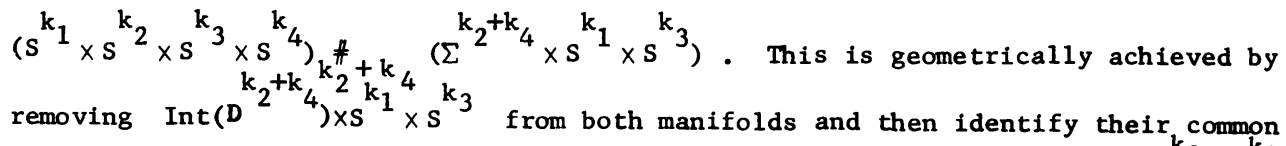
boundary ${ }_{k_{2}} \mathrm{k}_{4}$ Thus nothing else other than taking the usual conpected sum of $s^{k_{2}} \times s^{k_{4}}$ and $\Sigma^{2^{+k} / 4}$ by removing the interior of an embedded disc $D^{+k_{4}}$ from each manifold and identify the manifolds along their common boundary $s^{k_{2}+k_{4}-1}$ to obtain $s^{k_{2}} \times s^{k_{4}} \# \Sigma^{k_{2}+k_{4}}$. This is a well-defined operation. We then take the cartesian product with $s^{k_{1}} \times s^{k_{3}}$ to have $s^{k_{1}} \times s^{k_{3}} \times\left(s^{k_{2}} \times s_{4} \# \Sigma^{k_{2}+k_{4}}\right)=$ $s^{k_{1}} \times s^{k_{3}} \times s^{k_{2}} \times s^{k_{4}} k_{k_{2}} \sum^{k_{2}+k_{4}} \times s^{k_{1}} \times s^{k_{3}}$. But $s^{k_{1}} \times s^{k_{3}} \times s^{k_{2}} \times s^{k_{4}}$ is diffeomorphic

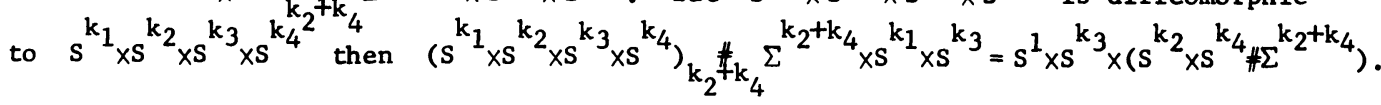
We will then prove the following.

CLASSIFICATION THEOREM If $\mathrm{M}^{\mathrm{n}}$ is a smooth manifold homeomorphic to $s^{k_{1}} \times s^{k_{2}} \times \ldots \times s^{k_{r}}$ where $2 \leq k_{1}<\ldots<k_{r-1}$ and $k_{4}-3 \leq k_{k}-1+k_{2}+k_{3} k_{r}$ and $n=k_{1}+k_{2}+\ldots+k_{r}$ then there exists homotopy spheres $\Sigma^{r-1} \sum_{1}+\ldots \Sigma_{2}^{-1} k_{3}, \ldots, \Sigma^{n-k_{1}}, \Sigma^{n}$ such that $M^{\mathrm{n}}$ is diffeomorphic to

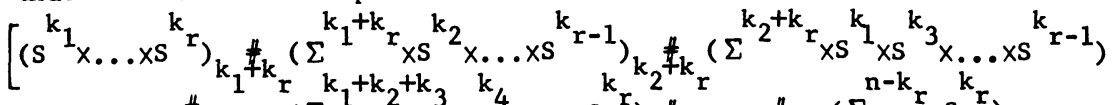

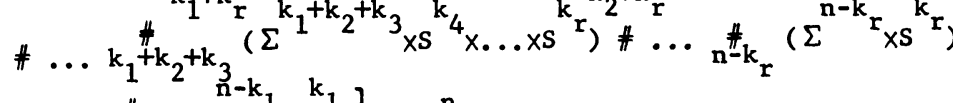

$$
\begin{aligned}
& \left.\# \ldots{ }_{n-k_{1}}^{\#}\left(\Sigma^{n-k_{1}}{ }^{*} s_{1}^{1}\right)\right] \# \Sigma^{n} \text {. }
\end{aligned}
$$

We shall use the above classification theorem to give the number of differentiable structures on $s^{k_{1}} s^{k_{2}} x_{\ldots} \ldots s^{k_{r}}$. We shall lastly compute the number of structures in some simple cases.

\section{PRELTMINARY RESULTS}

We shall apply obstruction theory of Munkres [4]. Let $M$ and $N$ be smooth $n$ manifolds and $L$ a closed subset of $M$ when triangulated. A homeomorphism $f: M \rightarrow N$ is a diffeomorphism modulo $L$ if $f \mid(M-L)$ is a diffeomorphism and each simplex $\alpha$ of $L$ has a neighborhood $V$, such that $f$ is smooth on $V-L$ near $\alpha$. By [4], if two n-manifolds $M$ and $N$ are combinatorially equivalent then $M$ is diffeomorphic modulo an $(n-1)$-skeleton $L$ onto $N$.

If $f: M^{n} \rightarrow N^{n}$ is a diffeomorphism modulo m-skeleton $m<n$ then Munkres showed that the obstruction to deforming $f$ to a diffeomorphism $8: M^{n} \rightarrow N^{n}$ modulo $(m-1)$-skeleton is an element $\lambda_{m}(f) \in H_{m}\left(M, \Gamma^{n-m}\right)=\Gamma^{n-m}$. Where $\Gamma^{n-m}$ is a group of diffeomorphisms of $\mathrm{s}^{\mathrm{n}-\mathrm{m}-1}$ modulo the diffeomorphisms that are extendable to diffeomorphisms of $D^{n-m}$. We call $g$ the smoothing of $f$. If $\lambda_{m}(f)=0$ then $g$ exists. Recall that in ([1], Lemma 2.1.1) we proved that if $q \geq p$ then $\Sigma^{p} \times S^{q}$ is diffeomorphic to $s^{p} \times s^{q}$ for any homotopy sphere $\Sigma^{p}$. In Remark (1) following that lemma, we showed further that even when $p-3 \leq q$ the result is still true.

LEMMA 2.1 Suppose $f: M^{n} \rightarrow s^{k_{1}} \times s^{k} x_{\ldots} \ldots s^{k}$ is a piecewise linear homeomorphism which is a diffeomorphism modulo $\left(n-k_{i}\right)$-skeleton $1 \leq 1 \leq r$, then there exists an 
homotopy sphere $\Sigma^{k} i$ and a piecewise linear homeomorphism

$$
h: M^{n} \rightarrow s^{k_{1}} \times s^{k_{2}} \times \ldots \times s^{k_{i-1}} \times \Sigma^{k_{i}} \times s^{k_{i+1}} \times \ldots \times s^{k} r
$$

which is a diffeomorphism modulo $\left(n-k_{i}-1\right)$ skeleton.

PROOF. Since $f: M^{n} \rightarrow s^{k_{1}} \times \ldots \times s^{k}$ is a diffeomorphism modulo $\left(n-k_{i}\right)$ skeleton then by Munkres [4], the obstruction to deforming $f$ to a diffeomorphism modulo $\left(n-k_{i}-1\right)-s k e l e t o n$ is an $k_{k_{i}-1} k_{i}-1 \lambda_{i}(f) \in H_{n-k_{i}}\left(M^{n}, \Gamma^{k_{i}}\right)=\Gamma^{k_{i}}$. Let $[\psi]=\lambda_{k}(f) \in \Gamma^{k_{i}}$ where $\psi: s^{k_{i}-1} \rightarrow s^{k_{i}-1}$ is a diffeomorphism. We define $\Sigma^{k_{i}}=D_{1}^{k_{i}^{i}} \cup_{\Downarrow} D_{2}^{k_{i}}$ and a homeomorphism $j: s^{k_{i}} \rightarrow \Sigma^{k_{i}}$ where we have $s^{k_{i}}=$ $D_{1}^{k_{i}} \cup D_{2} k_{i d}^{\psi}$ and so $j$ is identity map on $\operatorname{Int}\left(D_{1}^{k_{i}}\right)$ and radial extension of $\psi^{-1}$ on $\mathrm{D}_{2}{ }^{\mathrm{i}}$. So $\mathrm{j}$ is a piecewise linear homeomorphism by the definition and the obstruction to deforming $j$ to a diffeomorphism is $\left[\psi^{-1}\right]=-\lambda_{k_{i}}$ (f). So consider the map

$$
i d \times j:\left(s^{k_{1}} \times \ldots \times s^{k_{i}-1} \times s^{k_{i}+1} \times \ldots \times s^{k}\right) \times s^{k_{i}} \rightarrow\left(s^{k_{1}} \times \ldots \times s^{k_{i}^{-1}} \times s^{k_{i}+1} \times \ldots \times s^{k}\right) \times \Sigma^{k_{i}}
$$

The map is a piecewise linear homeomorphism and the obstruction to deforming ${ }_{k_{i}} f^{t}$ to $a_{k_{k}}$ diffeomorphism is $\left[\psi^{-1}\right]=-\lambda_{k_{i}}(f)$. Notice that the manifold $\left(s^{k_{1}} \times \ldots \times s^{k_{i}} \times \ldots \times s^{k_{r}}\right)$ $\times s^{k_{1}}=s^{k_{1}} \times \ldots \times s^{k_{i-1}} \times s^{k_{i}} \times s^{k_{i+1}} \times \ldots \times s^{k_{r}}$ and $\left(s^{k_{1}} \times \ldots \times s^{k_{i-1}} \times s^{k_{i+1}} \times \ldots \times s^{k_{r}}\right) \times$

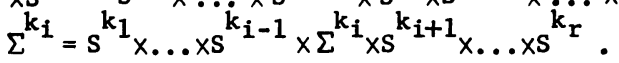

Consider the composite $(i d x j) \cdot f=h$, the obstruction to deforming $h$ to a diffeomorphism modulo $\left(n-k_{i}-1\right)$ skeleton $\underset{k_{1}}{i s} \lambda_{k_{i}}\left(\underset{k_{i}-1}{(h)}=\lambda_{k_{i}}((i d x j) \cdot f)=\lambda_{k_{i}}(i d x j)+\lambda_{k_{j}}(f)\right.$ $=-\lambda_{k_{i}}(f)+\lambda_{k_{i}}(f)=0$ hence $h: M^{n} \rightarrow s^{k_{1}} \times \ldots \times s{ }^{1-1} \times \Sigma^{k_{i}} \times s^{k_{i+1}} \times \ldots \times s$ is a diffeomorphism modulo $\left(n-k_{i}-1\right)$ skeleton. Hence the lemma.

LEMMA 2.2 Let $f: M^{n} \rightarrow s^{k_{1}} \times \ldots \times s^{k_{r}}$ be a diffeomorphism modulo $n-\left(k_{i}+k_{j}\right)$ skeleton $1 \leq i, j \leq r$ then there exists homotopy sphere $\Sigma^{k_{i}+k_{j}}$ and a piecewise linear homeomorphism

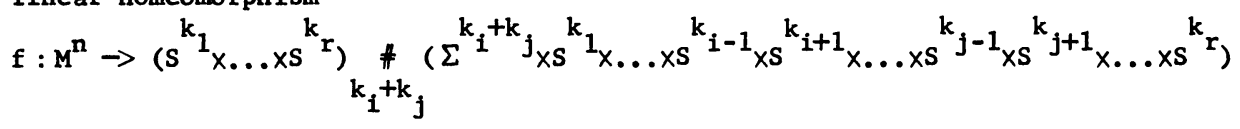

which is a diffeomorphism modulo $n-\left(k_{i}+k_{j}\right)-1$ skeleton.

PROOF. Since $f$ is a diffeomorphism modulo $n-\left(k_{i}+k_{j}\right)$ skeleton, it follows that the obstruction to deforming $f$ to a diffeomorphism modulo $n-\left(k_{i}+k_{j}\right)-1$ skeleton is

$$
\lambda(f) \in H_{n-\left(k_{i}+k_{j}\right)}\left(M^{n}, \Gamma^{k_{i}+k_{j}}\right)=\Gamma^{k_{i}+k_{j}} \text {. Let }[\phi]=\lambda(f) \in \Gamma^{k_{i}+k_{j}}
$$

where $\phi: s^{k_{1}+k_{j}-\frac{1}{1}} \rightarrow s^{k_{i}+k_{j}-1}$ is a diffeomorphism and $\Sigma^{k_{i}+k_{j}}=D^{k_{i}+k_{j}} \cup D^{k_{i}+k_{j}}$. We define $j: s^{k_{i}} \times s^{k_{j}} \rightarrow s^{k_{i}} \times s^{k_{j}} \# \Sigma^{k_{i}+k_{j}}$ to be identity map on $s^{k_{i}} \times s^{k_{j}} \stackrel{\phi}{1 n t(D}^{\left.k_{1}+k_{j}\right)}$ and radial extension of $\phi^{-1}$ on $\operatorname{Int}\left(D^{k_{i}+k_{j}}\right)$ hence $j$ is a piecewise linear homeomorphism and the obstruction to deforming $j$ to a diffeomorphism is $\left[\phi^{-1}\right]=-\lambda(f)$. Then consider 


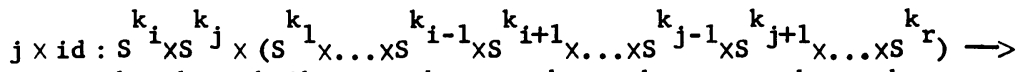

$$
\begin{aligned}
& \left(s^{k^{i}} \times s^{k_{j}} \# \Sigma^{k_{i}+k_{j}}\right) \times\left(s^{k_{1}} \times \ldots \times s^{k_{i-1}} \times s^{k_{i+1}} \times \ldots \times s^{k_{j-1}} \times s^{k_{j+1}} \times \ldots \times s^{k}\right) \text {. }
\end{aligned}
$$

Note that

$$
\begin{aligned}
& s^{k_{i}} \times s^{k_{j}} \times\left(s^{k_{1}} \times \ldots \times s^{k_{i}-1} \times s^{k_{i}+1} \times \ldots \times s^{k_{j-1}} \times s^{k_{j+1}} \times \ldots \times s^{k^{k}}\right)=
\end{aligned}
$$

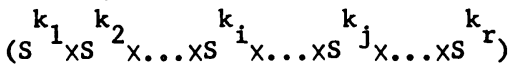

and

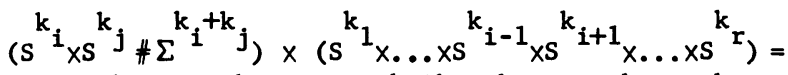

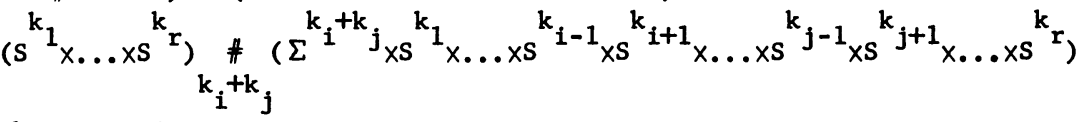

hence the above map is

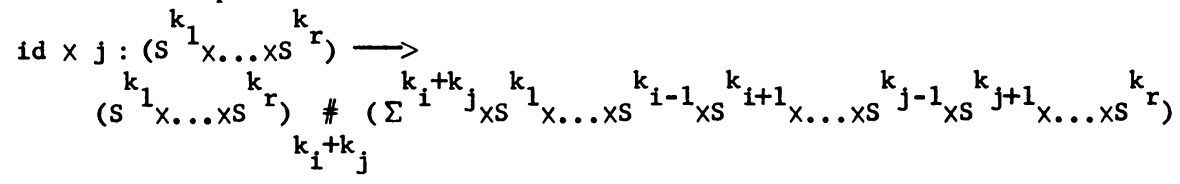

which is piecewise linear and its obstruction to a diffeomorphism is $-\lambda(f)$ hence the obstruction to deforming the composite $(j \times i d) \cdot f$ to a diffeomorphism modulo $n-\left(k_{i}+k_{j}\right)-1$ skeleton is zero. Hence if $f^{\prime}=(j \times i d) \cdot f$ then $f^{\prime}: M^{n} \longrightarrow$

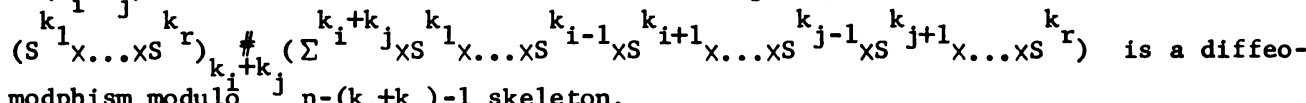
modphism modulo $n-\left(k_{i}+k_{j}\right)-1$ skeleton.

\section{CLASSIFICATION}

THEOREM 3.1 If $M^{n}$ is a smooth manifold homeomorphic to $s^{k}{ }^{k} S^{k}{ }^{k} \ldots . . . S^{k} r$ then there exists homotopy spheres, $\Sigma^{k_{1}+k_{r}}, \Sigma^{k_{2}+k_{r}}, \ldots, \Sigma^{k_{1}+k_{2}+k_{3}}, \ldots, \ldots \Sigma^{n-k} r, \ldots$ $\Sigma^{n-k} 1$, and $\Sigma^{n}$ such that $M^{n}$ is diffeomorphic to

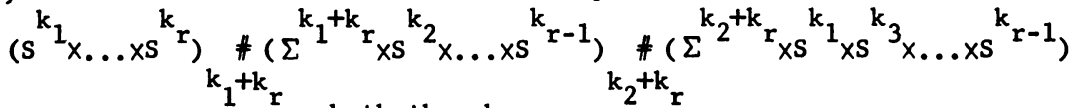

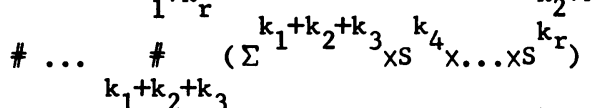

$$
\begin{aligned}
& \ldots{\underset{n-k}{n}}^{\#}\left(\Sigma^{n-k_{r}} \times s^{k}\right)_{n-k_{1}}^{\#}\left(\Sigma^{n-k_{1}} \times s^{k}\right) \# \Sigma^{n}
\end{aligned}
$$

where $2 \leq k_{1}<k_{2}<\ldots<k_{r}, k_{r}-3 \leq k_{r-1} \leq k_{r}$ and $n=k_{1}+k_{2}+\ldots+k_{r} \cdot$ PROOF. Suppose $M^{n} \stackrel{h}{\longrightarrow} s^{k_{1}} x_{.} . . x S^{k_{r}}$ is the homeomorphism. By Munkres theory [4], $h$ is a diffeomorphism modulo $(n-1)$ skeleton. Since the first non-zero homology appears in dimension $n-k_{1}$, (apart from the zero dimension) it then means that $h$ is a diffeomorphism modulo $\left(n-k_{1}\right)$ skeleton. The obstruction to deforming $h$ to a diffeomorphism modulo $\left(n-k_{1}-1\right)$ skeleton is $\lambda(h) \in H_{n-k_{1}}\left(M^{n}, \Gamma^{k_{1}}\right)=\Gamma^{1}$. By Lemma 2.1, there exists a piecewise linear homeomorphism $h^{\prime}$ and a homotopy sphere $\Sigma^{k^{1}}$ such that $h^{\prime}: M^{n} \rightarrow \Sigma^{k_{1}} \times s^{k_{2}} \times \ldots \times s^{k_{r}}$ which is a diffeomorphism modulo $\left(n-k_{1}-1\right)$ 
skeleton. In [1] Lemma 2.1.1 it was proved that $\Sigma^{k_{1}} \times s^{k_{2}}$ is diffeomorphic to $s^{k_{1}} \times s^{k_{2}}$ since $k_{1}<k_{2}$. It then follows that $\Sigma^{k_{1}} \times s^{k_{2}} \times \ldots \times s^{k_{r}}$ is diffeomorphic to $s^{k_{1}} \times \ldots \times s^{k_{r}}$ hence $h^{\prime}: M^{n} \rightarrow s^{k_{1}} \times \ldots \times s^{k_{r}}$ is a diffeomorphism modulo $\left(n-k_{1}-1\right)$ skeleton. There is no other obstruction to deforming $h^{\prime}$ to a diffeomorphism until the $\left(n-k_{2}-1\right)-s k e l e t o n$. This is because

$$
H_{i}\left(M^{n}, z\right)=0
$$

for $n-k_{2}+1<i<n-k_{1}$. So we can assume that $h^{\prime}$ is a diffeomorphism modulo $\left(n-k_{2}\right)$ skeleton. The obstruction to deforming $h^{\prime}$ to a diffeomorphism modulo $\left(n-k_{2}-1\right)$ skeleton is $\lambda\left(h^{\prime}\right) \in \mathrm{H}_{n-k_{2}}\left(M^{n}, \Gamma^{k_{2}}\right)=\Gamma^{k_{2}}$. Again by Lemma 2.1, there exists a homotopy sphere $\Sigma^{k_{2}}$ and a piecewise linear homeomorphism $h^{\prime \prime}: M^{n} \rightarrow s^{k_{1}} \times \Sigma^{k_{2}} \times$ $s^{k_{3}} \times \ldots \times s^{k_{r}}$ which is a diffeomorphism modulo $\left(n-k_{2}-1\right)$ skeleton. By the same argument as above since $k_{2}<k_{3}$ we see that $\sum^{k_{2}} \times s_{3}$ is diffeomorphic to $s^{k_{2}} \times s^{k_{3}}$ hence $s^{k_{1}} \times \Sigma^{k_{2}} \times s^{k_{3}} \times \ldots \times s^{k_{r}}$ is diffeomorphic to $s^{k_{1}} \times s^{k_{2}} \times s^{k_{3}} \times \ldots \times s^{k}$. This shows that $h^{\prime \prime}: M^{n} \rightarrow s^{1} X_{\ldots} \ldots S^{k_{r}}$ is a diffeomorphism modulo (n-k $2^{-1)-s k e l e t o n}$. By the same argument since $M^{n}$ has no homology between $n-k_{3}-1$ and $n-k_{2}-1$ we can assume that $h^{\prime \prime}$ is a diffeomorphism modulo $\left(n-k_{3}\right)$-skeleton. Proceeding this way using the same argument we can construct a homeomorphism say $h^{\prime \prime}: M^{n} \rightarrow s^{k_{1}} \times \ldots \times s^{k_{r}}$ which is a diffeomorphism modulo $\left(n-k_{r}\right)$-skeleton. However, to deform $h^{\prime \prime}$ to a diffeomorphism modulo $\left(n-k_{r}-1\right)$-skeleton, there is an obstruction $\lambda\left(h^{\prime \prime}\right) \underset{k_{r}}{\epsilon} H_{n-k_{r}}\left(M^{n}, \Gamma^{k_{r}}\right)=$ $\Gamma^{k_{r}}$. It also follows by Lemma 2.1 that there exists a homotopy sphere $\Sigma^{k_{r}}$ and a piecewise linear homeomorphism $f: M^{n} \rightarrow s^{k_{1}} x_{\ldots} \ldots s^{k_{-1}} \times \Sigma^{k_{r}}$ which is a diffeomorphism modulo (n-k $\left.{ }_{r}-1\right)$-skeleton. Now in Remark (1) of [1] it was shown that even when $\mathrm{p}-3 \leq \mathrm{r}, \mathrm{s}^{\mathrm{r}} \times \Sigma^{\mathrm{p}}$ is diffeomorphic to $\mathrm{s}^{\mathrm{r}} \times \mathrm{s}^{\mathrm{p}}$ and so by our assumption that $k_{r}-3 \leq k_{r-1} \leq k_{r}$ it follows that $s^{k_{r-1}} \times \Sigma^{k_{r}}$ is diffeomorphic to $s^{k_{r-1}} \times s^{k_{r}}$. Hence $\mathrm{s}^{\mathrm{k}_{1}} \times \ldots \times \mathrm{s}^{\mathrm{k}_{\mathrm{r}}-1^{\mathrm{r}} \times \Sigma^{\mathrm{k}} \mathrm{r}}$ is diffeomorphic to $\mathrm{s}^{\mathrm{k}_{1}} \times \ldots \times \mathrm{s}^{\mathrm{k}_{\mathrm{r}-1}} \times \mathrm{s}^{\mathrm{k}_{\mathrm{r}}}$ and so $\mathrm{f}: \mathrm{M}^{\mathrm{n}} \rightarrow$ $s^{k_{1}} \times \ldots \times s^{k_{r}}$ is a diffeomorphism modulo $\left(n-k_{r}-1\right)-s k e l e t o n$. The next obstruction to deforming $f$ to a diffeomorphism is on $n-\left(k_{r}+k_{1}\right)-1$ skeleton and it is $\lambda(f) \in H_{n-\left(k_{1}+k_{r}\right)}\left(M^{n}, \Gamma^{k_{1}+k_{r}}\right)=\Gamma^{k_{1}+k_{r}}$. By Lemma 2.2, there exists a piecewise linear homeomorphism

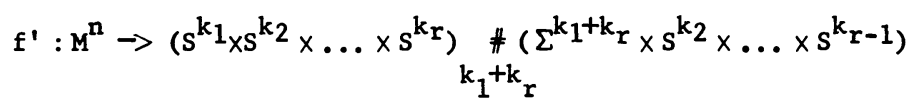

which is a diffeomorphism modulo $n-\left(k_{1}+k_{r}\right)-1$ skeleton for some homotopy sphere $\Sigma^{k_{1}+k_{r}}$ defined using $\lambda(f) \in \Gamma^{k_{1}+k_{r}}$. At this point, we want to remark that if $k_{1}+k_{r}-3 \leq \max \left(k_{2}, \ldots, k_{r-1}\right)$ and suppose $k_{j}=\max \left(k_{2}, \ldots, k_{r-1}\right)$ then it follows from Remark (1) of [1] since $k_{1}+k_{r_{1}} \leq k_{j}$, that $\Sigma^{k_{1}+k_{r}} \times s^{k_{j}}$ is diffeomorphic to $s^{k_{1}+k_{r}} \times s^{k_{j}}$ and so $\Sigma^{k_{1}+k_{r}} \times s^{k_{2}} \times \ldots \times s^{k_{r-1}}$ is diffeomorphic to $s^{k_{1}+k_{r}} \times s^{k_{1}} \times \ldots \times s^{k_{r-1}}$.

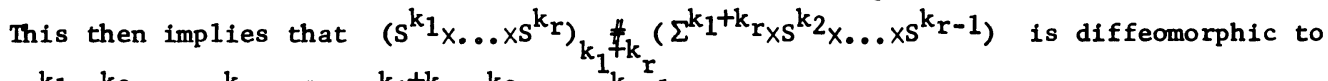

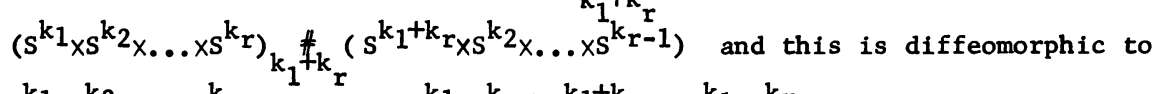
$s^{k_{1}} \times s^{k_{2}} \times \ldots \times s^{k_{r}}$ because $s^{k_{1}} \times s^{k_{r}} \# s^{k_{1}+k_{r}}=s^{k_{1}} x^{k_{r}}$. So this means that the factor 
$\Sigma^{k_{1}+k_{r}} \times s^{k_{2}} \times \ldots \times s^{k_{r}-1}$ will disappear in the above sum if we have the condition $k_{1}+k_{r}-3 \leq \max \left(k_{2}, \ldots, k_{r-1}\right)$.

Anyway, we have $f^{\prime}: M^{n} \rightarrow\left(s^{k} x_{\ldots} \ldots \times s^{k_{r}}\right)_{k_{1}+k_{r}}^{\#}\left(\Sigma^{k} 1^{+k} \times s^{k_{2}} \times \ldots \times s^{k} r-1\right)$ which is a diffeomorphism modulo $n-\left(k_{1}+k_{r}\right)-1$ skeleton. Since $H_{i}\left(M^{n}, z\right)=0$ for $n-\left(k_{2}+k_{r}\right)<$ $i \leq n-\left(k_{1}+k_{r}\right)-1$ then there is no obstruction to deforming $f^{\prime}$ to a diffeomorphism modulo $n-\left(k_{2}+k_{r}\right)$ skeleton and the obstruction to deforming $f^{\prime}$ to a diffeomorphism modulo $n-\left(k_{2}+k_{r}\right)-1$ skeleton is $\lambda\left(f^{\prime}\right) \in H_{n-\left(k_{2}+k_{r}\right)}\left(M^{n}, \Gamma^{\left.k_{2}+k_{r}\right)}=\Gamma^{k_{2}+k_{r}}\right.$. Using the same technique as in the proof of Lemma 2.2 it can be easily shown that there exists an homotopy sphere $\Sigma^{k_{2}+k_{r}}=D^{k_{2}+k_{r}} \bigcup^{k_{2}+k_{r}}$ where $\psi=\lambda\left(f^{\prime}\right) \in \Gamma^{k_{2}+k_{r}}$ and $\psi: s^{k_{2}+k_{r}-1} \rightarrow s^{k_{2}+k_{r}-1}$ is a diffeomorphism and a piecewise linear homeomorphism

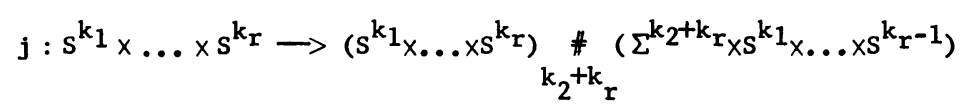

where obstruction to a diffeomorphism is $-\lambda\left(f^{\prime}\right)$. We now define a map

$$
\begin{aligned}
& j^{\prime}:\left(s^{k} x_{\ldots} \ldots s^{k_{r}}\right)_{k_{1}+k_{r}}^{\#}\left(\Sigma^{k_{1}+k^{k}} r_{x} s^{k_{2}} x \ldots x s^{k_{r}-1}\right) \\
& \longrightarrow\left(s^{k_{1}} \times \ldots \times s^{k_{r}}\right)_{k_{2}+k_{r}}^{\#}\left(\Sigma^{k_{2}+k} r_{x} s^{k_{1}} 1_{x} s^{k_{3}} \times \ldots \times s^{k_{r}-1}\right) \underset{k_{1}+k_{r}}{\#}\left(\Sigma^{k_{1}+k} r_{x s^{k}} k_{2} \ldots \times s^{k_{r}-1}\right)
\end{aligned}
$$

where $j^{\prime}=j$ on $\left(s^{k_{1}} \times \ldots \times s^{k} r\right)-\operatorname{Ind}\left(D^{k_{1}+k}\right) \times s^{k_{2}} \times \ldots \times s^{k} r-1$ and identity on $\Sigma^{k_{1}+k_{r}} \times s^{k_{2}} \times \ldots \times s^{k_{r}-1}-\operatorname{Int}\left(D^{k_{1}+k_{r}}\right) \times s^{k_{2}} \times \ldots \times s^{k_{r-1}}$.

Clearly $j^{\prime}$ is piecewise linear and its obstruction to a diffeomorphism is

$-\lambda\left(f^{\prime}\right)$ hence the obstruction to deforming the composite $g=j^{\prime} \cdot f^{\prime}$ where

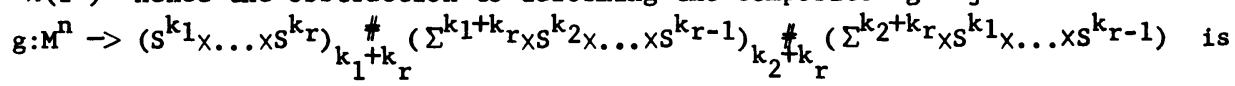
$\lambda\left(j^{\prime} \cdot f^{\prime}\right)=\lambda\left(j^{\prime}\right)+\lambda\left(f^{\prime}\right)=0$. Hence $g=j^{\prime} \cdot f^{\prime}$ is a diffeomorphism modulo $n-\left(k_{2}+k_{r}\right)-1$ skeleton. Proceeding in this way, we see that the next obstruction to a diffeomorphism will be on $\left(n-\left(k_{3}+k_{r}\right)\right)$-skeleton. Using the above technique continuously, we can construct a piecewise linear homeomorphism

$$
\begin{aligned}
& g^{\prime}: M^{n} \rightarrow\left(s^{k_{1}} x_{\ldots} \ldots \times s^{k_{r}}\right) \underset{k_{1}+k_{r}}{\#}\left(\Sigma^{k_{1}+k_{r}} \times s^{k_{2}} \times \ldots x s^{k_{r}-1}\right)
\end{aligned}
$$

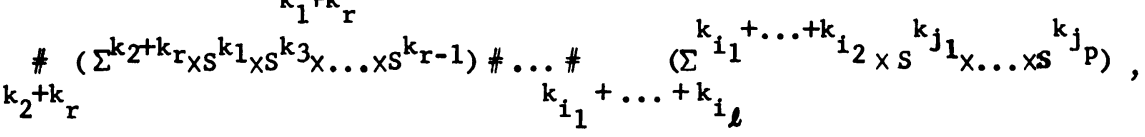

$$
\begin{aligned}
& j_{p}^{\prime} s \neq i_{\ell}
\end{aligned}
$$

which is a diffeomorphism modulo $n-\left(k_{r-1}+\ldots+k_{1}\right)=k_{r}$ skeleton. The obstruction to extending $g^{\prime}$ to a diffeomorphism modulo $\left(k_{r}-1\right)$ skeleton is $\lambda\left(g^{\prime}\right) \in H_{k_{r}}\left(M^{n}, \Gamma^{n-k_{r}}\right)=$ $\Gamma^{n-k_{r}}$. By using the same technique as in the proof of Lemma 2.1 , there exists a piecewise linear homeomorphism $j$ and homotopy sphere $\Sigma^{n-k_{r}}$ such that

$$
j: s^{k_{1}} \times \ldots \times s^{k_{r}} \longrightarrow\left(s^{k_{1}} \times \ldots \times s^{k_{r}}\right) \underset{n-k_{r}}{\#}\left(\Sigma^{n-k_{r}} \times s^{k_{r}}\right)
$$

has an obstruction to a diffeomorphism to be ${ }^{r}-\lambda\left(g^{\prime}\right)$. From this we define the map, 


$$
\begin{aligned}
& j^{\prime}:\left(s^{k_{1}} \times \ldots \times s^{k_{r}}\right)_{k_{1}+k_{r}}^{\#}\left(\Sigma^{k_{1}+k_{r}} \times s^{k_{2}} \times \ldots \times s^{k_{r}-1}\right)
\end{aligned}
$$

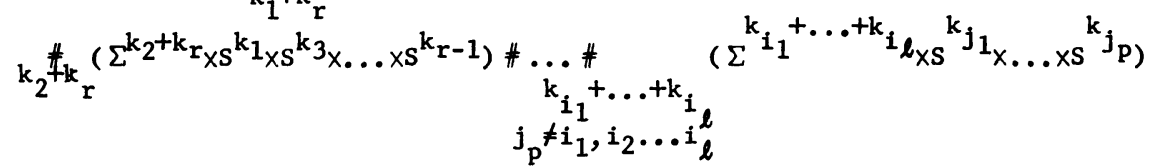

$$
\begin{aligned}
& \rightarrow\left(s^{k_{1}} x_{\ldots} \ldots s^{k_{r}}\right)_{n-k_{r}}^{\#}\left(\Sigma^{n-k_{r}} r_{x} s^{k_{r}}\right)_{k_{1}+k_{r}}^{\#}\left(\Sigma^{k_{1}+k_{r_{x}}} s^{k_{2}} \times \ldots \times s^{k_{r}-1}\right) \\
& \text { \#...\# }\left(\Sigma^{k_{i_{1}}+\ldots+k_{i} \ell} \times s^{k_{j_{1}}} \times \ldots \times s^{k_{j}}\right) \\
& \mathrm{k}_{\mathrm{i}_{1}}+\ldots+\mathrm{k}_{\mathrm{i}_{\ell}}
\end{aligned}
$$

where $j^{\prime}=j$ on $\left(s^{k_{1}} x_{\ldots} \ldots s^{k} r\right)-\left(\operatorname{Int}\left(D^{k_{1}+k}\right) \times s^{k} x_{\ldots} \ldots s^{k_{r}-1}\right)$ and identity elsewhere. It is easily seen that $j^{\prime}$ is piecewise linear homeomorphism and the obstruction to deforming the composite $j^{\prime} \cdot g^{\prime}$ to a diffeomorphism is zero. Hence the map $h^{\prime}=j^{\prime} \cdot g^{\prime}$ where

$$
\begin{aligned}
& h^{\prime}: M^{n} \rightarrow\left(s^{k} 1_{x} \ldots \times s^{k_{r}}\right)_{k_{1} t_{k}}\left(\Sigma^{k} 1^{+k_{r}} \times s^{k_{2}} \times \ldots \times s^{k_{r}-1}\right) \\
& \underset{k_{2}+k_{r}}{\#}\left(\Sigma^{k_{2} 2^{+k_{r}}} \times s^{k_{1}} 1_{x} s^{k_{3}} \times \ldots x s^{k_{r}-1}\right) \# \ldots \underset{n-k_{r}}{\#}\left(\Sigma^{n-k_{r}} \times s^{k_{r}}\right)
\end{aligned}
$$

is a diffeomorphism modulo $\left(k_{r}-1\right)$ skeleton. However, since $H_{i}\left(M^{n}, z\right)=0$ for $k_{r-1}<i<k_{r}-1$, there is no more obstruction to deforming $h^{\prime}$ to a diffeomorphism modulo $k_{r-1}$-skeleton. To deform $h^{\prime}$ to a diffeomorphism modulo $\left(k_{r-1}-1\right)$ skeleton, there is an obstruction and this equals $\lambda\left(h^{\prime}\right) \in H_{k_{r-1}}\left(M^{n}, \Gamma^{n-k_{r-1}}\right)=\Gamma^{n-k_{r-1}}$. Applying the above technique again, we can get an homotopy sphere $\sum^{n-k_{r-1}}$ and a piecewise linear homeomorphism

$$
\begin{aligned}
& h^{\prime \prime}: M^{n} \rightarrow\left(s^{k} x_{\ldots} \ldots s^{k_{r}}\right)_{k_{1}+k_{r}}^{\#}\left(\Sigma^{k_{1}+k_{r}} x_{x s^{k}} x_{\ldots x} \ldots s^{k_{r}-1}\right) \\
& \left.\underset{k_{2}+k_{r}}{\#}\left(\Sigma^{k_{2}+k_{r}} \times s^{k_{1}} \times s^{k_{3}} \times \ldots x s^{k_{r}-1}\right) \underset{k_{1}+k_{2}+k_{3}}{\ldots} \underset{\left(\sum^{k}+k_{2}+k_{3}\right.}{\ldots} \times s^{k_{4}} \times \ldots \times s^{k_{r}}\right) \\
& \cdots n_{n-k_{r}}^{\#}\left(\Sigma^{n-k_{r}} \times s^{k_{r}}\right) \underset{n-k_{r-1}^{*}}{*}\left(\Sigma^{n-k_{r-1}} \times s^{k_{r}-1}\right)
\end{aligned}
$$

which is a diffeomorphism modulo $k_{r-1}-1$ skeleton. The next obstruction will be on $k_{r-2}-1$ skeleton. Proceeding this way gradually down the remaining skeleton, we can construct a map

$$
\begin{aligned}
& g: M^{n} \rightarrow\left(s^{k} x_{\ldots} \ldots s^{k^{k}}\right)_{k_{1}+k_{r}}^{\#}\left(\Sigma^{k_{1}+k_{r}} \times s^{k_{2}} \times \ldots \times s^{k_{r}-1}\right)
\end{aligned}
$$

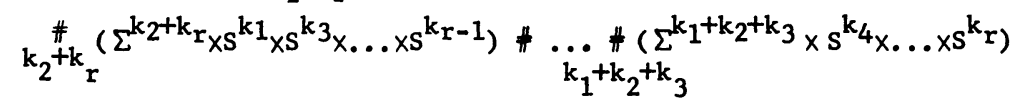

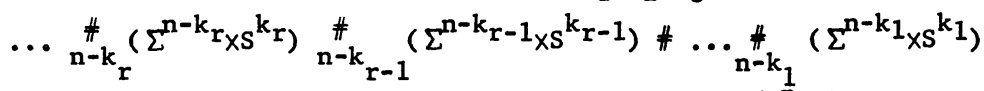

which is a diffeomorphism modulo $k_{1}$-skeleton. Since $H_{i}\left(M^{\frac{1}{n}}, z\right)=0$ for $0<i<k$, then $\mathrm{g}$ is a diffeomorphism modulo one point. It therefore follows that there exist an homotopy sphere $\Sigma^{n}$ such that $M^{n}$ is diffeomorphis to 


$$
\begin{aligned}
& {\left[\left(s^{k_{1}} x_{\ldots} \ldots s^{k_{r}} \underset{k_{1}+k_{r}}{\#}\left(\Sigma^{k_{1}+k_{r}} \times s^{k_{2}} \times \ldots x s^{k_{r}-1}\right) \underset{k_{2}+k_{r}}{\#}\left(\Sigma^{k_{2}+k} \times s^{k_{1}} 1_{x} s^{k_{3}} \times \ldots x s^{k_{r}-1}\right)\right.\right.}
\end{aligned}
$$

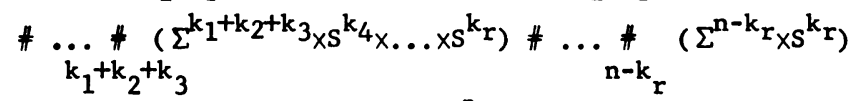

$$
\begin{aligned}
& \left.\# \ldots \underset{n-k_{1}}{\#}\left(\Sigma^{n-k_{1}} \times s^{k_{1}}\right)\right] \# \Sigma^{n} \text {. }
\end{aligned}
$$

Hence the theorem.

Recall that $H(p, k)$ denotes the subgroup of $\theta^{p}$ consisting of homotopy $p$-spheres $\Sigma^{\mathrm{p}}$ such that $\Sigma^{\mathrm{p}} \times \mathrm{s}^{\mathrm{p}}$ is diffeomorphic to $\mathrm{s}^{\mathrm{p}} \times \mathrm{s}^{\mathrm{k}}$.

THEOREM 3.2 The number of differentiable structures on $s^{k_{1}} \times \ldots \times s^{k_{r}}$ where $2 \leq k_{1}<k_{2}<\ldots<k_{r-1}$ and $k_{r}-3 \leq k_{r-1} \leq k_{r}$ equals the order of the group

$$
\begin{aligned}
& \frac{\theta^{k_{1}+k_{r}}}{H\left(\left(k_{1}+k_{r}\right),\left(k_{3}, \ldots, k_{r-1}\right)\right)} \times \frac{\theta^{k_{2}+k_{r}}}{H\left(k_{2}+k_{r},\left(k_{1}, k_{3}, \ldots, k_{r-1}\right)\right)} \times \ldots \times \\
& \frac{\theta^{k_{1}+k_{2}+k_{3}}}{H\left(\left(k_{1}+k_{2}+k_{3},\left(k_{4}, \ldots, k_{r}\right)\right)\right.} \times \ldots \times \frac{\theta^{n-k_{r}}}{H\left(n-k_{r}, k_{r}\right)} \times \ldots \times \frac{\theta^{n-k_{1}}}{H\left(n-k_{1}, k_{1}\right)} \times \theta^{n} .
\end{aligned}
$$

PROOF Let $\left(0\left(k_{1}+k_{r}\right), 0\left(k_{2}+k_{r}\right), \ldots, 0\left(k_{1}+k_{2}+k_{3}\right), \ldots, 0\left(n-k_{r}\right), \ldots, 0\left(n-k_{1}\right), 0(n)\right)$ represent the trivial elements of $\theta^{k_{1}+k_{r}}, \theta^{k_{2}+k_{r}}, \ldots, \theta^{k_{1}+k_{2}+k_{3}}, \ldots, \theta^{n-k_{1}}, \theta^{n}$, then we define a map

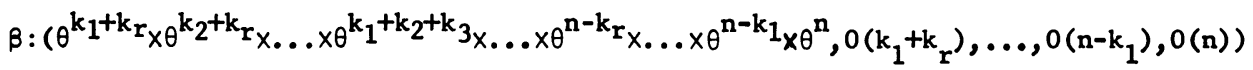

$$
\longrightarrow \text { (structures on } s^{k_{1}} \times \ldots \times s^{\left.k_{r}, 0\right)}
$$

where 0 represents the usual structures on $s^{k_{1}} x_{\ldots \times} \ldots s^{k_{r}}$. If $\Sigma^{k_{1}+k_{r}} \in \theta^{k_{1}+k_{r}}$, $\ldots, \Sigma^{k_{1}+k_{2}+k_{3}} \in \theta^{k_{1}+k_{2}+k_{3}}, \ldots, \Sigma^{n-k_{r}} \in \theta^{n-k_{r}}, \ldots, \Sigma^{n-k_{1}} \in \theta^{n-k_{1}}$ and $\Sigma^{n} \in \theta^{n}$ then we define

$$
\begin{aligned}
& \beta\left(\Sigma^{k_{1}+k_{r}}, \Sigma^{k_{2}+k_{r}}, \ldots, \Sigma^{k_{1}+k_{2}+k_{3}}, \ldots, \Sigma^{n-k_{r}}, \ldots, \Sigma^{n-k_{1}}, \Sigma^{n}\right)= \\
& {\left[\left(s^{k} x_{\ldots} \ldots s^{k_{r}}\right) \underset{k_{1}+k_{r}^{\#}}{\#}\left(\Sigma^{k_{1}+k_{r}} \times s^{k_{2}} \times \ldots \times s^{k_{r}-1}\right)_{k_{2}+k_{r}}^{\#}\left(\Sigma^{k_{2}+k_{r}} s^{k_{1}} s^{k_{3}} \times \ldots \times s^{k_{r}-1}\right)\right.} \\
& \text { \#... } \underset{\mathrm{k}_{1}+\mathrm{k}_{2}+\mathrm{k}_{3}}{*}\left(\Sigma^{\mathrm{k}_{1}+\mathrm{k}_{2}+\mathrm{k}_{3}} \times \mathrm{s}^{\mathrm{k}_{4}} \times \ldots \times s^{\mathrm{k}_{\mathrm{r}}}\right) \# \underset{\mathrm{n}-\mathrm{k}_{\mathrm{r}}}{\#}\left(\Sigma^{\mathrm{n}-\mathrm{k}_{\mathrm{r}}} \times s^{\mathrm{k} r}\right) \\
& \left.* \ldots \text { * }_{n-k_{1}}^{*}\left(\Sigma^{n-k_{1}} \times s^{k_{1}}\right)\right] * \Sigma^{n} \text {. }
\end{aligned}
$$

$\beta$ is we11-defined because if

$$
\begin{aligned}
& \Sigma_{1}^{k_{1}+k_{r}}, \Sigma_{2}^{k_{1}+k_{r}} \in \theta^{k_{1}+k_{r}} ; \Sigma_{1}^{k_{2}+k_{r}}, \Sigma_{2}^{k_{2}+k_{r}} \in \theta^{k_{2}+k_{r}} ; \ldots \Sigma_{1}^{k_{1}+k_{2}+k_{3}}, \Sigma_{2}^{k_{1}+k_{2}+k_{3}} \in \\
& \theta^{k_{1}+k_{2}+k_{3}} \ldots ; \Sigma_{1}^{n-k_{r}}, \Sigma_{2}^{n-k_{r}} \in \theta^{n-k_{r}} ; \ldots ; \Sigma_{1}^{n-k_{1}}, \Sigma_{2}^{n-k_{1}} \in \theta^{n-k_{1}} ; \Sigma_{1}^{n}, \Sigma^{n} \in \theta^{n}
\end{aligned}
$$

are $h$-cobordant respectively then they are diffeomorphic. It then follows that $\Sigma_{1}^{k_{1}+k_{r}} \times s^{k_{2}} \times \ldots \times s^{k_{r}-1}$ is diffeomorphic to $\Sigma_{2}^{k_{1}+k_{r}} \times s^{k_{2}} \times \ldots \times s^{k_{r}-1}$ and $\Sigma_{1}^{k_{2}+k_{r}} \times s^{k_{1}} \times s^{k_{3}} \times \ldots \times s^{k_{r}-1}$ is diffeomorphic to $\Sigma_{2}^{k_{1}+k_{r}} \times s^{k_{1}} \times s^{k_{3}} \times \ldots \times s^{k_{r}-1}$ and 
$\Sigma_{1}^{k_{1}+k_{2}+k_{3}} \times s^{k_{4}} \times s^{k_{5}} \times \ldots \times s^{k_{r}}$ is diffeomorphic to $\Sigma_{2}^{k_{1}+k_{2}+k_{3}} \times s^{k_{4}} \times \ldots \times s^{k_{r}}$. Also $\Sigma_{1}^{n-k_{r}} \times s^{k_{r}}$ is diffeomorphic to $\Sigma_{2}^{n-k_{r}} \times s^{k_{r}}$ and $\Sigma_{1}^{n-k_{1}} \times s^{k_{1}}$ is diffeomorphic to $\Sigma_{2}^{n-k_{1}} \times s^{k_{1}}$ and so this means that

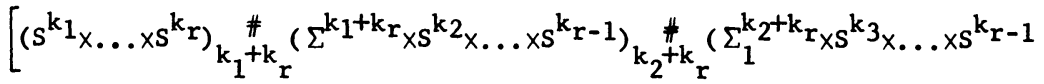

$$
\begin{aligned}
& \text { \#.... } k_{1}+k_{2}^{*}+k_{3}\left(\Sigma_{1}^{k_{1}+k_{2}+k_{3}} \times s^{k_{4}} \times \ldots \times s^{k_{r}}\right) \# \ldots \\
& \text { \#.... } \left.{ }_{n-k_{r}}^{\#}\left(\Sigma_{1}^{n-k_{r}} \times s^{k} r\right) \# \ldots n_{n-k_{1}}^{\#}\left(\Sigma_{1}^{n-k_{1}} s^{k}{ }_{1}\right) \# \Sigma_{1}^{n}\right] \text { is diffeomorphic to }
\end{aligned}
$$

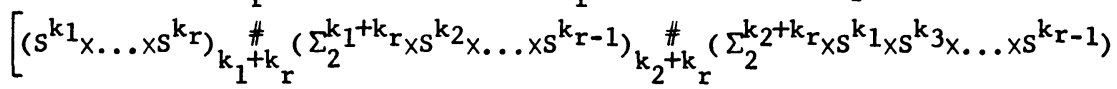

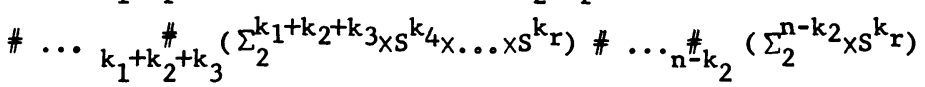

$$
\begin{aligned}
& \left.\# \ldots \mathrm{n}_{\mathrm{n}-\mathrm{k}_{1}}^{\#}\left(\Sigma_{2}^{\mathrm{n}-\mathrm{k}_{1}} \mathrm{~s}^{\mathrm{k}_{1}}\right)\right] \# \Sigma_{2}^{\mathrm{n}} \text {. }
\end{aligned}
$$

Hence $\beta$ is well-defined map.

Clearly $\beta$ takes the base points $0\left(k_{1}+k_{r}\right), 0\left(k_{2}+k_{r_{1}}\right), \ldots, 0\left(k_{1}+k_{2}+k_{3}\right), \ldots, 0\left(n-k_{r}\right)$, $\ldots, O\left(n-k_{1}\right), O(n)$ to the base point 0 . This is because if all the homotopy spheres $\Sigma^{k} s$ are standard spheres, then all the summands involving $\Sigma^{i} s$ in the image of $\beta$ will vanish leaving only $s^{k} 1 \times \ldots \times s^{k} r$. By Theorem $3.1, \beta$ is onto.

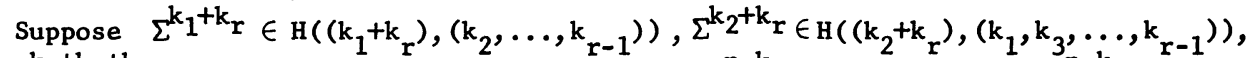

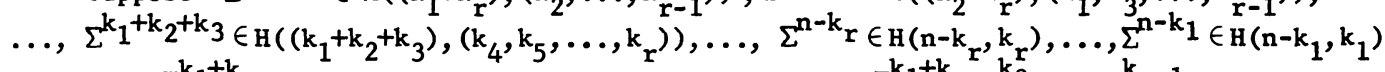
then for $\Sigma^{k_{1}+k_{r} \in H\left(\left(k_{1}+k_{r_{1}}\right),\left(k_{2}, \ldots, k_{r-1}\right)\right)}$ this means $\Sigma^{k_{1}+k_{r \times s} k_{2} \times \ldots \times s} k_{r-1}$ is diffeomorphic to $s^{k_{1}+k^{k}} r_{x} s^{k_{2}} \times \ldots \times s^{k_{r-1}}$ hence $s^{k_{1}} 1_{x} \ldots \times s^{k_{r}} \# \Sigma^{k_{1}+k} r_{x} s^{k_{2}} \times \ldots \times s^{k-1}$ is

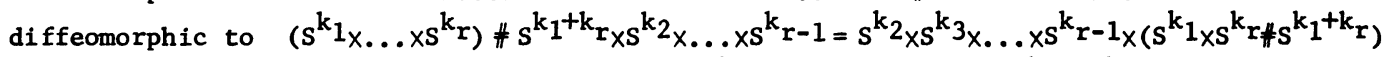
which is diffeomorphic to $s^{k_{2}} \times s^{k_{3}} \times \ldots \times s^{k} r-1_{X} s^{k} 1_{X} s^{k}$ since $s^{k_{1}} \times s^{k} r \# s^{k} 1^{+k_{r}}=$

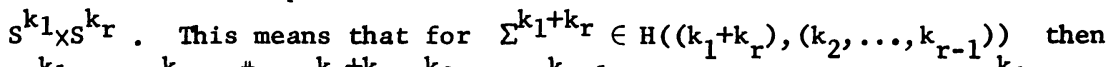
$\left(s^{k} 1_{x} \ldots \times s^{k}\right)_{k_{1}+k_{r}}^{\#}\left(\Sigma^{k_{1}+k} \times s^{k}{ }^{k} \ldots \times s^{k} r-1\right)$ is diffeomorphic to $s^{k_{1}} 1_{x} \ldots \times s^{k} r$ and so the summand $\Sigma^{k_{1}+k} r_{x} k_{2} \times \ldots \times s^{k_{r-1}}$ in the image $\beta$ vanishes. Similar arguments show that all other summands involving the $\Sigma^{i} s$ in $H(i, n-i)$ vanish hence in this case

$$
\beta\left(\Sigma^{k_{1}+k_{r}}, \Sigma^{k_{2}+k_{r}}, \ldots, \Sigma^{k_{1}+k_{2}+k_{3}}, \ldots, \Sigma^{n-k_{r}}, \ldots, \Sigma^{n-k_{1}}, \Sigma^{n}\right)=s^{k_{1}} \times s^{k_{2}} \times \ldots \times s^{k_{r}}
$$

Then $\beta$ induces a map.

$$
\begin{gathered}
\Phi:\left(\frac{\theta^{k_{1}+k_{r}}}{H\left(k_{1}+k_{r},\left(k_{2}, \ldots, k_{r-1}\right)\right)} \times \frac{\theta^{k_{1}+k_{2}}}{H\left(k_{2}+k_{r},\left(k_{1}, k_{2}, \ldots, k_{r-1}\right)\right)} \times \ldots \times\right. \\
\frac{\theta^{k_{1}+k_{2}+k_{3}}}{H\left(k_{1}+k_{2}+k_{3},\left(k_{4}, \ldots, k_{r}\right)\right)} \times \ldots \times \frac{\theta^{n-k_{r}}}{H\left(n-k_{r}, k_{r}\right)} \times \ldots \times \frac{\theta^{n-k_{1}}}{H\left(n-k_{1}, k_{1}\right)} \\
\left.\times \theta^{n}\right) \rightarrow\left(s t r u c t u r e s \text { on } s^{k_{1}} \times \ldots \times s^{\left.k_{r}\right)}\right.
\end{gathered}
$$

which is on to since $\beta$ is onto.

$$
\text { If } \Phi\left(\Sigma^{k_{1}+k_{r}}, 0\left(k_{2}+k_{r}\right), \ldots, 0\left(k_{1}+k_{2}+k_{3}\right), \ldots, 0\left(n-k_{r}\right), \ldots, 0\left(n-k_{1}\right), 0(n)\right)=0 \text { then }
$$
it follows by an easy generalization of Theorem 2.2.1 of [1] that $\Sigma^{k_{1}+k_{r} \in H\left(\left(k_{1}+k_{r}\right),\left(k_{2}, \ldots, k_{r-1}\right)\right)}$ and by the same method if $\Phi\left(0\left(k_{1}+k_{r}\right), 0\left(k_{2}+k_{r}\right)\right.$, $\left.\left.\ldots, \Sigma^{n-k_{r}}\right), \ldots, \Sigma^{n-k_{r}}, \ldots, O\left(n-k_{1}\right), O(n)\right)=0$ then $\Sigma^{n-k_{r} \in H\left(n-k_{r}, k_{r}\right)}$. Also in 
[[5], Theorem A], Reinhard Schultz showed that the inertial group of product of any number of ordinary spheres is trivial. This result implies that if $\Phi\left(0\left(k_{1}+k_{r}\right)\right.$, $\left.0\left(k_{2}+k_{r}\right), \ldots, 0\left(n-k_{r}\right), \ldots, 0\left(n-k_{1}\right), \Sigma^{n}\right)=0$ then $\Sigma^{n}$ is diffeomorphic to $s^{n}$. It then follows that $\Phi$ is one to one and onto hence the number of differentiable structures on $s^{k_{1}} \times s^{k}{ } \times \ldots \times s^{k} r$ is equal to the order of

$$
\begin{gathered}
\frac{\theta^{k_{1}+k_{r}}}{H\left(k_{1}+k_{r},\left(k_{2}, \ldots, k_{r-1}\right)\right)} \times \frac{\theta^{k_{2}+k_{r}}}{H\left(k_{2}+k_{r}, k_{1}, k_{3}, \ldots, k_{r-1}\right)} \times \ldots \times \frac{\theta^{k_{1}+k_{2}+k_{3}}}{H\left(\left(k_{1}+k_{2}+k_{3}\right),\left(k_{4}, \ldots, k_{r}\right)\right)} \\
\ldots \times \frac{\theta^{k_{1}+k_{2}+k_{3}}}{H\left(\left(k_{1}+k_{2}+k_{3}\right),\left(k_{4}, \ldots, k_{r}\right)\right.} \times \ldots \times \frac{\theta^{n-k_{1}}}{H\left(n-k_{r}, k_{r}\right)} \times \ldots \times \frac{\theta^{n-k_{1}}}{H\left(n-k_{1}, k_{1}\right)} \times \theta^{n}
\end{gathered}
$$

\section{EXAMPLES}

We recal1 that in Table 7.4 of [5], $\theta_{k}^{n}$ denotes the number of homotopy spheres which do not embed in $\mathrm{R}^{\mathrm{n}+\mathrm{k}}$. We shall use the values computed in that table in some of the examples given here. Since $\Gamma^{i}=0$ for $1 \leq i \leq 6$, then the number of smooth structures on $s^{2} \times s^{2} \times s^{2} \times s^{2}$ is the order of $\theta^{8}=2$. Also since $\theta_{3}^{8}=2=\left|\theta^{8}\right|$ then $H(8,2)=0$ and so the number of smooth structures on $s^{2} \times s^{2} \times s^{2} \times s^{2} \times s^{2}=12$. By similar reasoning, the number of smooth structures on $s^{2} \times s^{2} \times s^{2} \times s^{4}=12$.

Since $\theta^{12}=0$ and $H(9,3)=4$ then the number of smooth structures on $s^{3} \times s^{3} \times S^{3} \times s^{3}=2$ whereas since $\theta^{15}=16256$ and $\theta^{9}=8$ combined with the fact that $\theta^{12}=0$ and $H(9,3)=4$ it follows that the number of smooth structures on $s^{3} \times s^{3} \times s^{3} \times S^{3} x s^{3}$ is 32512. From [3] we see that $\theta_{5}^{8}=1$ and $H(8,4)=\theta^{8}$ and $\Gamma^{12}=0$, then the number of smooth structures on $s^{4} \times s^{4} \times s^{4} \times s^{4}=2$. By a similar argument, it is easily seen that the number of smooth structures on $s^{4} x s^{4} x s^{4} x s^{4} x s^{4}$ is the order $\frac{\theta^{16}}{H(16,4)} \times \theta^{20}$. Also since $H(10,5)=\theta^{10}$ then the number of smooth structures on $s^{5} \times s^{5} \times s^{5} \times s^{5}$ is the order of $\frac{\theta^{15}}{H(15,5)} \times \theta^{20}$.

I am grateful to Professor P. Emery Thomas, University of California, Berkeley, for very useful communications.

\section{REFERENCES}

1. Samuel Omoloye Ajala. Differentiable structures on product of spheres, Houston Journal of Mathematics, Vol. 10, No. 1 (1984), 1-14.

2. R. de Sapio. Differentiable structures on product of spheres, Comment Math. Hal. (Vo1. 44), 1969.

3. D. Sullivan. On the Hauptvanmuting for manifolds, Bulletin American Math. Soc. 73 (1967), 598-600.

4. J. Munkres. Obstruction to smoothings of piecewise linear homomorphism, Annals of Math. 72 (1960), 521-554.

5. J. Levine. Classification of Differentiable knots, Annals of Math. 86 (1965), 15-50.

6. Reinhard Schultz. On inertial group of a product of spheres, Transactions of American Math. Soc. 156 (1971), 137-157. 


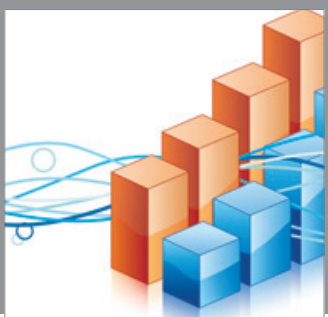

Advances in

Operations Research

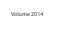

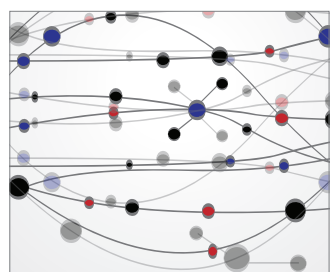

\section{The Scientific} World Journal
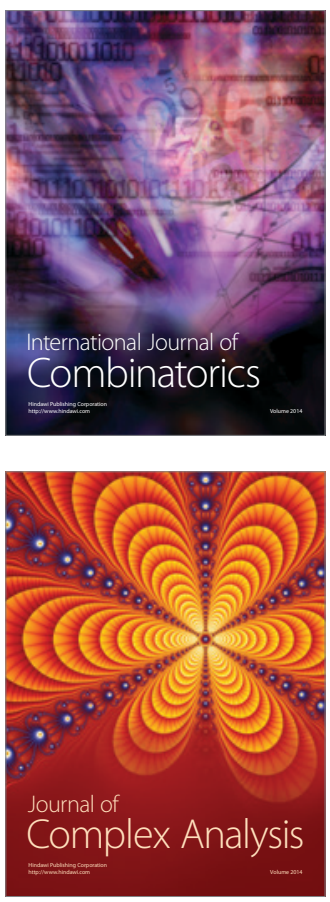

International Journal of

Mathematics and

Mathematical

Sciences
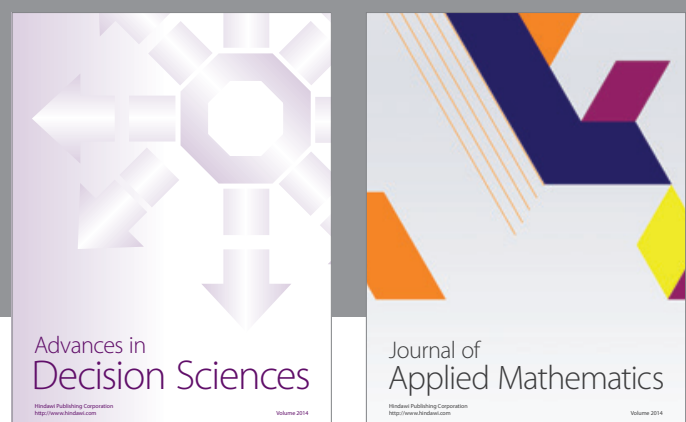

Journal of

Applied Mathematics
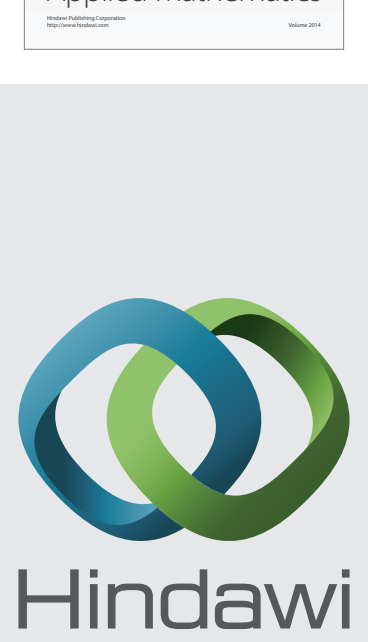

Submit your manuscripts at http://www.hindawi.com
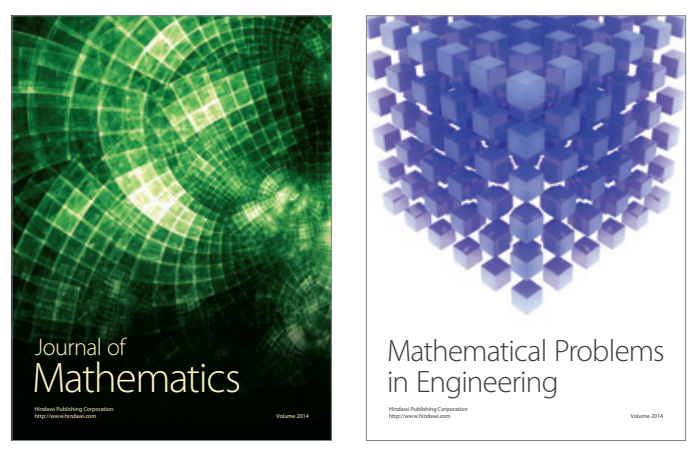

Mathematical Problems in Engineering
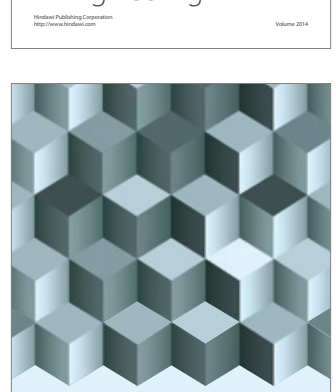

Journal of

Function Spaces
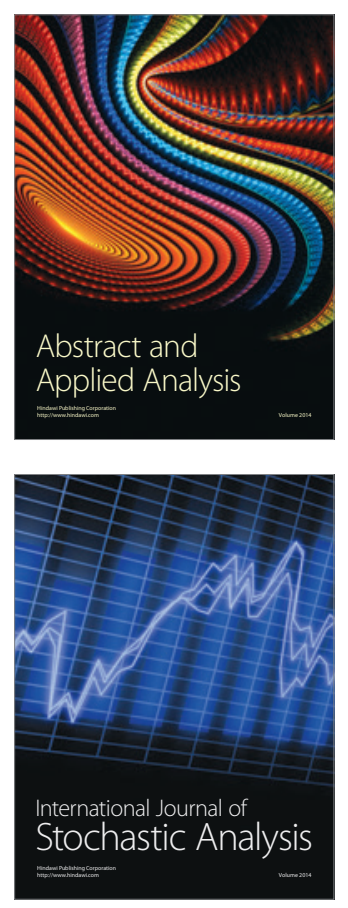

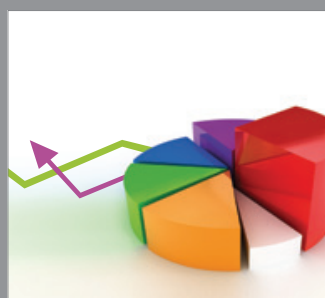

ournal of

Probability and Statistics

Promensencen
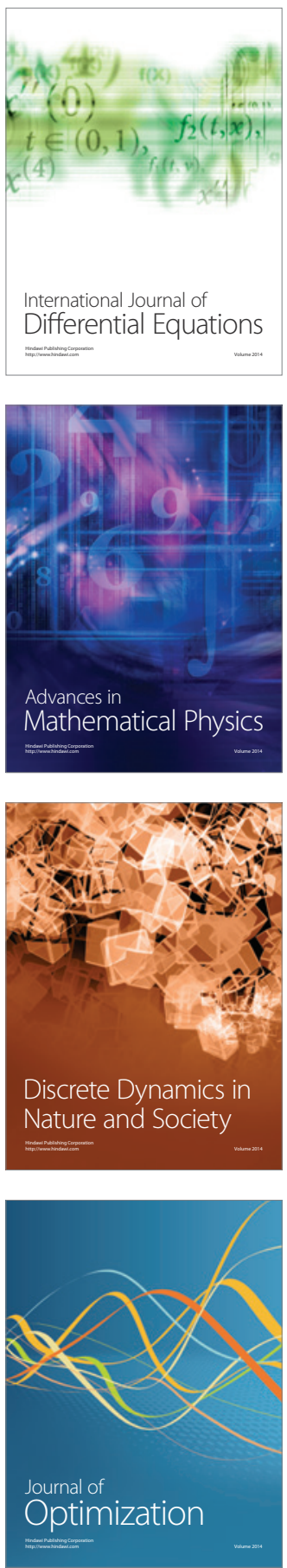